\title{
Digestion des constituants pariétaux et activité fermentaire cæcale chez le lapin en croissance : incidence du taux d'incorporation et de la granulométrie de la source de fibre
}

\author{
T Gidenne \\ INRA, laboratoire de recherche sur l'élevage du lapin, \\ BP 27, 31326 Castanet - Tolosan, Cedex, France
}

\begin{abstract}
Summary - Fibre digestion and cecal fermentation in the growing rabbit: effect of incorporation rate and degree of the fibre-source grinding. Fecal digestibility of the cell wall (CW) and levels of volatile fatty acids (VFA) in cecal contents were measured in growing rabbits. Increasing the level of $\mathrm{CW}$ from dehydrated lucerne (LD) increased $\mathrm{CW}$ degradation and levels of VFA, with a greater proportion of acetate. Fine grinding of LD (1 mm vs $3 \mathrm{~mm}$ ) did not affect CW digestibility or VFA level.
\end{abstract}

Chez le lapin, la dégradation des constituants pariétaux (CP) par la flore cæcale dépend de leur proportion par rapport aux glucides cytoplasmiques. Par ailleurs, la diminution de la taille des particules alimentaires stimule l'activité motrice antipéristaltique du côlon (Bouyssou et al, 1988) et pourrait accroître le temps de séjour des digesta et leur dégradation dans le cæcum. Les teneurs en acides gras volatils (AGV) cæcaux et la digestibilité apparente (CUD) ont été mesurées en fonction du taux d'incorporation et de la granulométrie d'une source unique de fibre : la luzerne déshydratée (LD).

Matériel et méthodes - Deux taux d'incorporation de LD et 2 grilles de broyage ont été comparés, soit 3 aliments : B $48,5 \%$,
$3 \mathrm{~mm} ; \mathrm{H} 76,5 \%, 3 \mathrm{~mm}$; F 76,5\%, $1 \mathrm{~mm}$. Ces aliments granulés (isoazotés) contenaient, en complément, de la farine de poisson et de l'amidon de maïs purifié; ils ont été distribués ad libitum à 3 lots de 12 lapereaux, âgés de 28 à 84 j (abattage). Les mesures de CUD ont été réalisées (6 lapins/lot) entre 70 et $84 j$ d'âge, puis les lapins ont été abattus et les contenus cæcaux prélevés en vue du dosage des AGV (Gidenne, 1986). Les CP ont été estimés selon le fractionnement de Van Soest aprés un prétraitement amylasique (Giger et al, 1979) et protéasique.

Résultats et discussion - Le taux ou la granulométrie de LD n'ont pas affecté la croissance des lapins, mais ont modifié significativement leur ingestion : $87,4 \pm 8,7^{\mathrm{a}} ; 114,1 \pm 7,7^{\mathrm{b}}$; $105,1 \pm 10,5^{b} \mathrm{~g} / \mathrm{j}$, respectivement pour 
les aliments $\mathrm{B}, \mathrm{H}$ et $\mathrm{F}$. L'élévation de la teneur en $\mathrm{CP}$ entre les aliments $\mathrm{B}$ $(23,6 \% \mathrm{NDF})$ et $\mathrm{H}(35,6 \% \mathrm{NDF})$ améliore significativement la digestibilité de la fraction NDF (+ $24 \%$ ), du fait d'une hausse du CUD de la fraction "hémicellulose (NDF ADF)" $(10,7$ vs $25,4 \%$ ), le CUD de la fraction ADF n'étant pas affecté (tableau I). Corrélativement, la teneur en AGV totaux du cæcum est alors plus élevée $(+70 \%)$ : hausse de l'acétate C2 (+ $59 \%$ ) et du propionate $\mathrm{C} 3(+60 \%)$, sans variation de la teneur en butyrate $\mathrm{C} 4$. Ainsi, la proportion de $\mathrm{C} 2$ passe de 73 à $80 \%$ entre les régimes $\mathrm{B}$ et $\mathrm{H}$; la proportion de $\mathrm{C} 4$ diminue (20 vs $13,7 \%$ ) et celle de C3 demeure inchangée $(6 \%)$.

Le broyage de la luzerne avec une grille de $1 \mathrm{~mm}$ augmente la proportion de particules de taille (T) inférieure à $0,3 \mathrm{~mm}(77,7$ vs $81,0 \%)$, et une disparition des particules grossières ( $T$ supérieure à $1 \mathrm{~mm}$, soit $1,5 \%$ dans le régime $H$ ). Le CUD des $C P$ du régime $F$ est équivalent à celui de $\mathrm{H}$; de même, les teneurs et les proportions des AGV cæcaux ne diffèrent pas significativement. Cette absence d'effet positif du broyage sur la dégradation des fibres, contrairement au résultats de Candau et al (1986), peut provenir de l'écart de granulométrie relativement faible entre les aliments $F$ et $H$, la LD étant initialement un produit relativement riche en fines particules (feuilles, granulation...).

En conclusion, l'élévation de la teneur en fibres provenant de luzerne a favorisé l'activité fermentaire cæcale et par conséquent, la dégradation des fibres. En revanche, le broyage de la luzerne n'a pas eu d'effet sensible sur la dégradation des constituants pariétaux.

Bouyssou T, Candau M, Ruckebusch $Y$ (1988) Reprod Nutr Dev 28, 181-182

Candau M, Auvergne A, Comes F, BouillierOudot M (1986) Ann Zootech 35, 373-386

Gidenne T (1986) Ann Zootech 35, 121-136

Giger $S$, Sauvant D, Dorleans $M$, MorandFehr P (1979) 30th Annual Meeting of the European Association for Animal Production, Harrogate, UK, July 1979

Tableau I. Digestibilité (\%) des constituants pariétaux et teneurs en acides gras volatils (mM/) cæcaux, en fonction du broyage et du taux d'incorporation de luzerne dans le régime.

\begin{tabular}{lcccccc}
\hline Régime & CUD NDF & CUD ADF & AGV totaux & Acétate & Propionate & Butyrate \\
\hline B & $14,7 \pm 2,8^{\mathrm{a}}$ & $15,8 \pm 2,8$ & $18,1 \pm 5,1^{\mathrm{a}}$ & $14,0 \pm 3,6^{\mathrm{a}}$ & $0,9 \pm 0,3^{\mathrm{a}}$ & $2,9 \pm 1,3$ \\
H & $18,2 \pm 3,3^{\mathrm{b}}$ & $15,9 \pm 3,6$ & $30,9 \pm 4,7^{\mathrm{b}}$ & $25,8 \pm 3,8^{\mathrm{b}}$ & $1,6 \pm 0,5^{\mathrm{b}}$ & $3,2 \pm 0,9$ \\
F & $18,9 \pm 3,0^{\mathrm{b}}$ & $16,1 \pm 3,5$ & $29,4 \pm 5,5^{\mathrm{b}}$ & $23,9 \pm 4,5^{\mathrm{b}}$ & $1,5 \pm 0,3^{\mathrm{b}}$ & $3,7 \pm 1,0$ \\
\hline
\end{tabular}

NDF : neutral detergent fiber; ADF : acid detergent fiber. 